\title{
Converting to terahertz communications
}

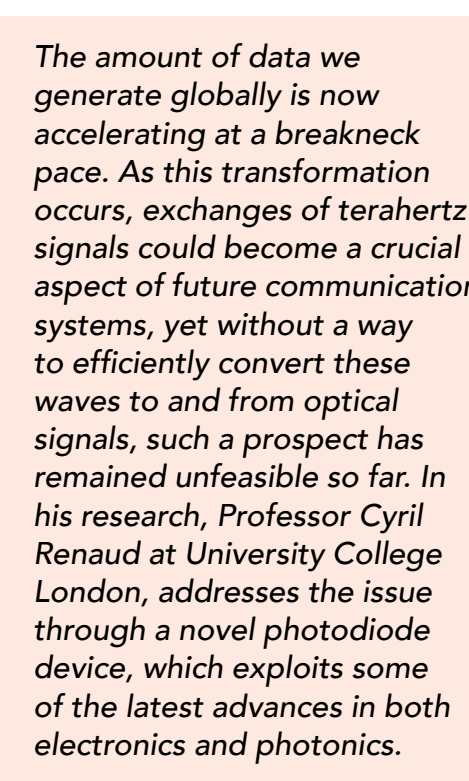

The amount of data we accelerating at a breakneck occurs, exchanges of terahert aspect of future communicatio waves to and from optical signals, such a prospect has his research Prible so far. Renaud at University College through a novel photodiode of the latest advances in both electronics and photonics. systems, yet without a way

ver the course of just a few
decades, the tools we use to communicate with each other have transformed almost beyon recognition, and for now, the pace of this change shows no signs of slowing down. As the technological landscap transforms around us, the amount of data generated globally is growing generated 130 exabytes of data in a single month for the first time information than was ever generated over a large majority of human history.

Such staggering growth has been strongly driven by the rise of wireless mobile devices, which have opened up internet access to many millions of people - particularly in the developing world. Inevitably, this is now putting an increasing strain on the wireless channels used to convey these signals to individual devices. Without new innovations, the infrastructures we rely on to communicate with each other could come under severe strain in the near future. In order to keep on top researchers and engineers are now aiming to accomplish higher rates of signal transmissions in wireless

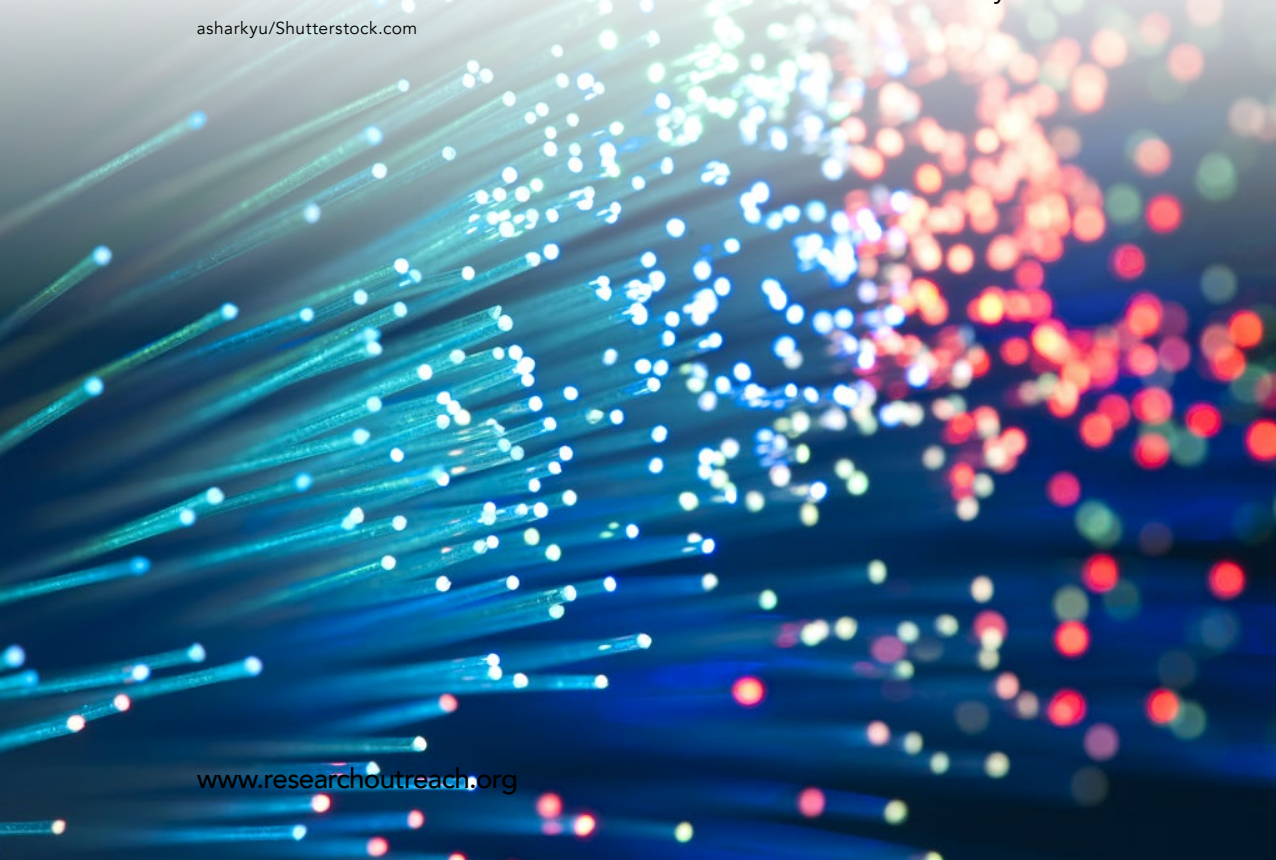

TERAHERTZ CARRIER FREOUENCIES One critically important aspect for researchers to consider in achieving this goal is the 'bandwidth' of wireless signals. The term describes the range of frequencies occupied by an information-carrying signal: with high bandwidths, individual signals can their specific frequencies. This creates a strong incentive for conveying terahertz frequencies - which fall between microwave and infrared frequencies on the electromagnetic spectrum. In the field of radio communications, these waves are the highest possible frequency at which signals can be transmitted.

With the bandwidths required by modern wireless communication systems now exceeding tens of gigahertz, such high frequencies are becoming increasingly desirable. Before systems can realistically operate at these frequencies, however, they through the abtacle. As they trave waves will readily interact with features ranging from water droplets to oxygen molecules, causing them to be scattered in unwanted directions. Ultimately, this makes them all but impossible to transmit over large distances, and entirely unsuitable for large-scale wireless networks.

\section{CONVERTING BETWEEN}

WAVELENGTHS

Professor Renaud proposes that this problem can be overcome by transmitting signals most of the way to their destinations using optical frequencies. Crucially, this can be don via well-established networks of fibre optic cables, which form the backbon
of modern internet infrastructures.

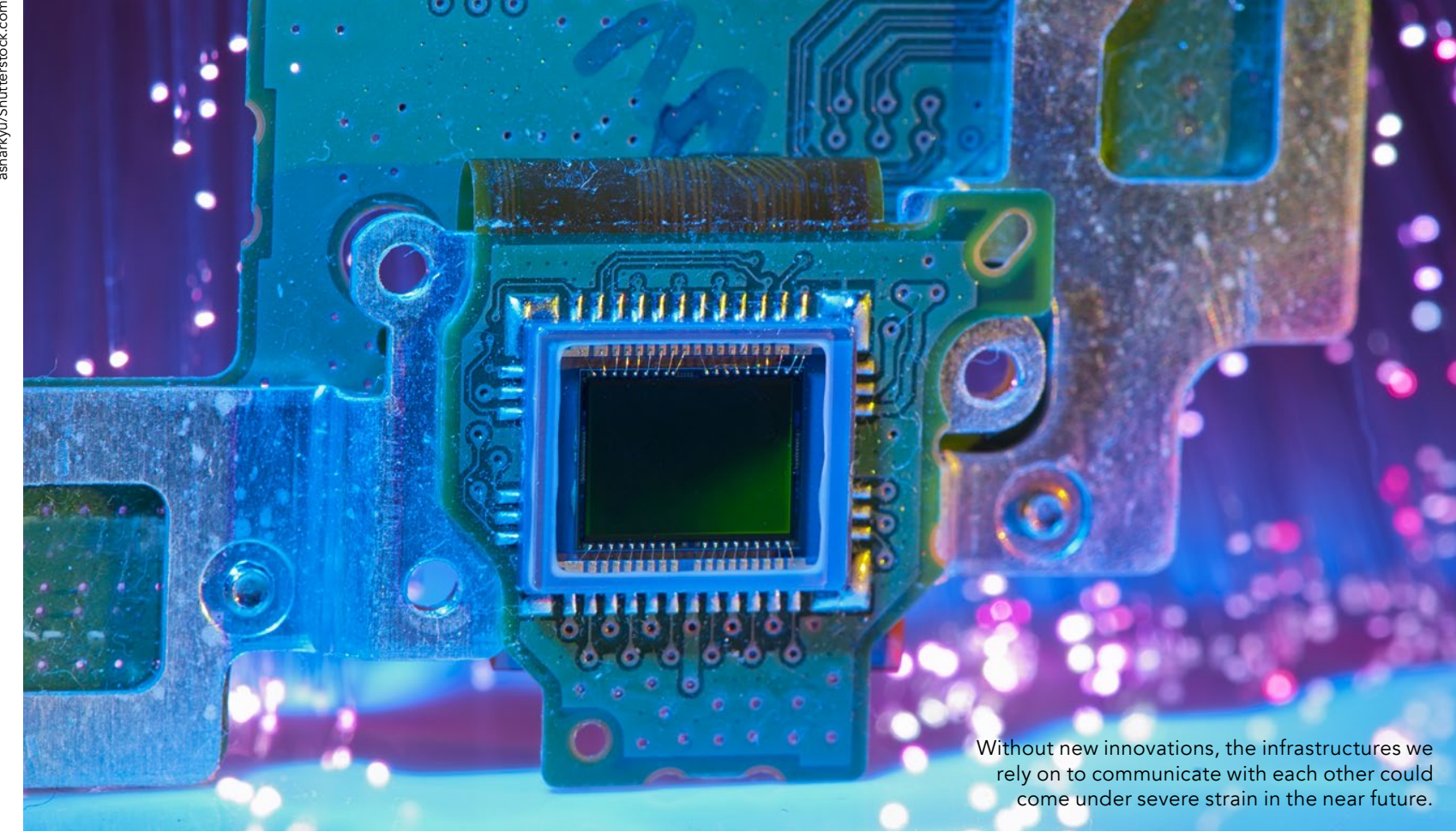

Once they are within the same building three semiconductors within an or local area as the receiving wireless applied electric field. Two of these device, these frequencies can then semiconductors are doped with. be converted into wireless terahertz signals, using an antenna. Finally, they can travel the last few tens or hundreds of metres to their destination, with little semiconductors are doped with different types of molecular impurity,

If response times are longer, the bandwidths which can be transmitted from the photodiode become lower - creating a trade-off between the giving them different properties: while sensitivity of the device and the amount
one acts as an electron donor, the
of information it can convey. So far, this one acts as an electron donor, the has made the devices unsuitable for the indoor teraherz communications

$\begin{array}{ll}\text { Such a system has } & \text { systems which } \\ \text { Professor Renaud }\end{array}$ Such a system has clear advantages, but there is still another hurdle to overcome: the conversion between signals
and electrical currents is not entirely efficient, With the bandwidths required by envisages. modern wireless communication systems IMPRoving now exceeding tens of gigahertz, PERORMANCE

high frequencies are becoming $\quad \begin{aligned} & \text { In his latest research, } \\ & \text { Professor Renaud }\end{aligned}$ increasingly desirable

meaning the information carried by contains no impurities. When an a signal can be lost in the process. Through his research, Professor Renaud addresses this problem through cutting-edge technologies, integrating principles from both electronics and photonics - a term describing how signals can be transmitted and recelved through the manipulation of light.

LIMITATIONS IN

REGULAR PHOTODIODES Converting light into an electrical curent requires a device named a 'photodiode'. These devices electron in this layer meets a photon higher energy level, and swept by the electric field to a positively charged cathode. In its place, a positively charged 'hole' is left behind, which is swept to a negatively charged anode. Altogether, this process generates a 'photocurrent' that can be used to generate an optical signal. However, this can only occur after a certain response time - describing the tim transit to their respective hole to has pioneered the Carrier PhotoDiodes' (UTC-PDs)-highspeed, high-output power devices which can overcome many of the issues faced by conventional photodiodes. Like their conventional counterparts, these photodiodes also contain three semiconductor materials in a linear the middle p. This time, however, doped with impurities which accept electrons, while the electron-accepting layer is left pure. When an electric field is applied to this arrangement, its spect the placements of mpuntes mean photons will respond extremely quickly. 


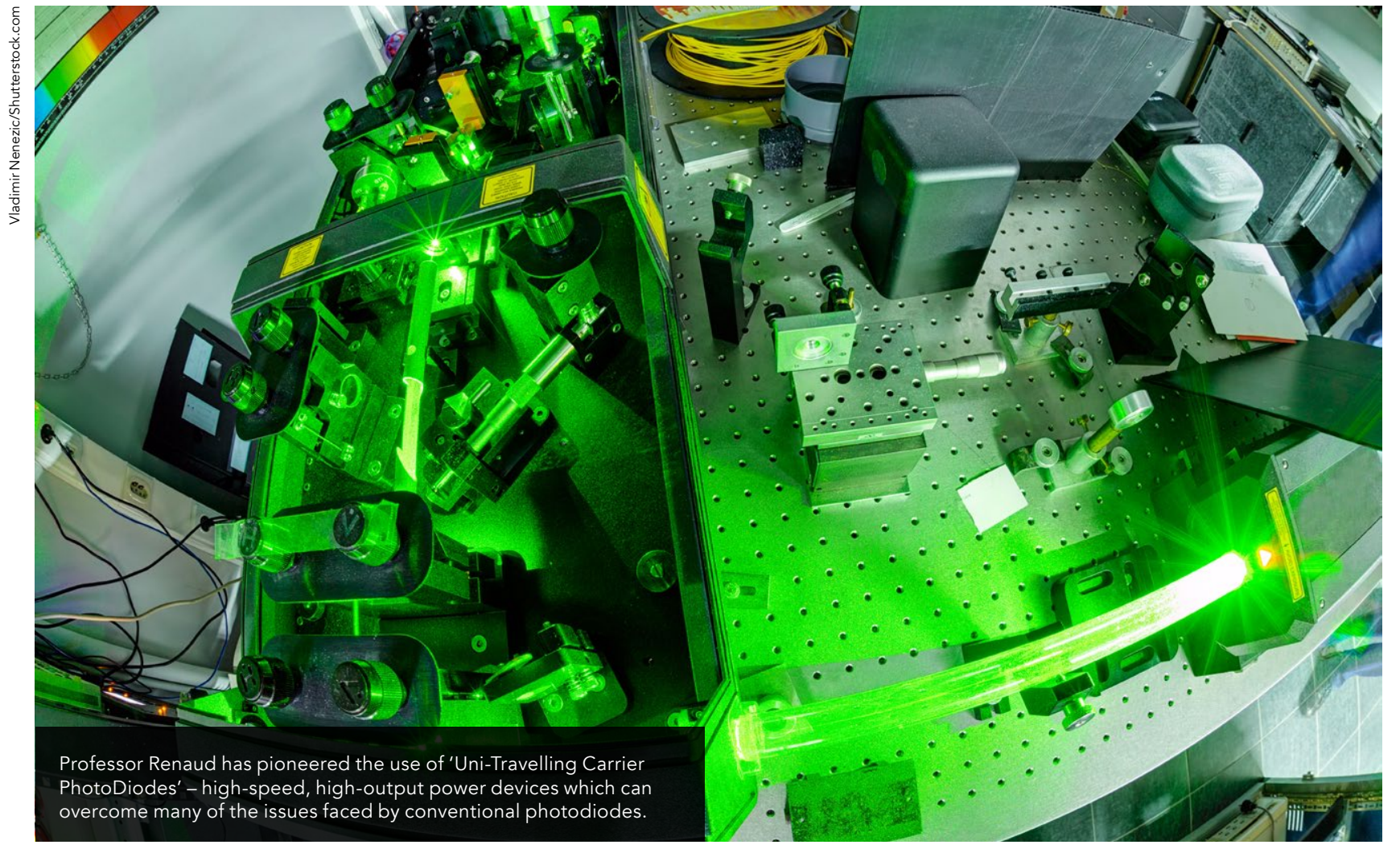
Where the overall photodiode response limiting the efficiency of conversion
time is mainly limited by the slower
between terahertz waves and electrical time is mainly limited by the slower current. The research team have transits of holes in conventiona arrangement in a UTC-PD means it is only limited by electron transport. In turn, the novel device reduces limitations on the bandwidth of the signal being converted, reducing the need for a trade-off between performance, an the amount of information to When applied to terahertz waves, this approach opens up new opportunities for communication systems which convey wireless, high-bandwidth signals over long distances.

\section{IMPROVING}

COMMUNICATION SYSTEMS Having demonstrated a strong basis for antennae which efficiently transmit now conceived a new approach to PDs, particularly when the devices are integrated with antennae.

Through their results, the researchers and receive terahertz signals, Profess Renaud's team have made significant progress towards indoor wireless networks incorporating the signals. If achieved, these networks would allow users of wireless devices to transmit and receive data ...these networks would allow users of than seemed wireless devices to transmit and receive possible until only data at far higher rates than seemed possible until only recently

Through future work, the research electrons and holes within UTC-PDs to further exploit cutting-edge enable significantly higher maximum principles of photonics to integrate output powers than those possible UTC-PDs with other elements of in conventional diodes. In turn, this photonic converters, including lasers,

UNPRECEDENTED OUTPUT POWER To demonstrate the promising capabilities of the UTC-PD, Professor Renaud and his colleagues have carried out a number of experiments to measure and assess their maximu output power. Typically, an upper limit is imposed on this value by a combination of thermal effects, and makes them ideal for converting high amplifiers, and modulators. In turn, frequencies like terahertz waves to high this would enable the team to carry degrees of efficiency. Furthermore, Professor Renaud and his colleagues - using devices the size of computer for the use UTC-PDs advantages chips. If their approach becomes more for the use of UTC-PDs in photomixing widely adopted, it could become a key where two laser beams with specific frequencies are overlapped, and focused onto a single device worldwide; ensuring our accelerating use of data can safely continue well into the future.

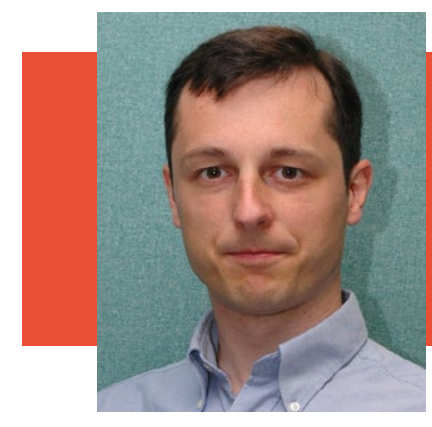

Behind the Research Professor Cyril Renaud

E: c.renaud@ucl.ac.uk
profile?upi=CRENA49

Research Objectives

Professor Renaud's research ranges from photonic devices to photonic system with particular interest photodiodes, integrated photonic, THz photonics,
sensor systems and wireless communication systems.

\section{Detail}

Cyril Renaud

Dept of Electronic and Electrical Engineering University College London

Torrington Place, London

WC1E 7JE, UK

Bio

Cyril C. Renaud received his Engineering degree from the Ecole Supérieure d'Optique, Orsay, France, and the Diplôme d'Etudes Approfondies (D.E.A.) in optics and photonics from the University Paris $X$, Orsay, 1996. He spent one year as a Project Engineer with Sfim-ODS, working on the development of microchips lasers and portable range finders. He then joined the Optoelectronics Research Centre, University of Southampton in 1998 to work on diode pumped highpower ytterbium-doped fibre-lasers, with particular interest on Q switched system and, with parm generation. Professor of photonics at Univerity College London, and Programme Director for the UCL/C a bidge Doctoral Training Centre in Connected Electronic and

Photonic Systems. He has published more than 200 per reviewed journals and international conferences, and three patents.

\section{Funding}

- EU commission

Collaborators

Professor Alwyn J. Seeds

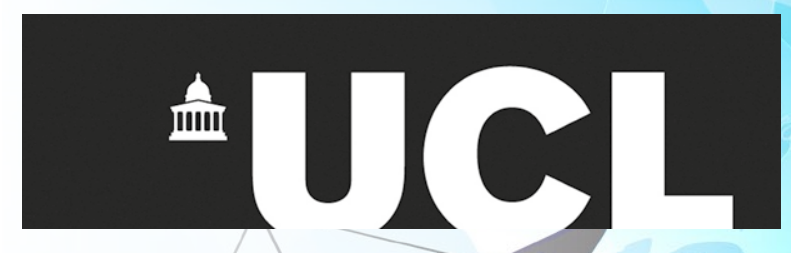

\section{References}

Renaud, C.C., Seddon, J., Graham, C., Lin, X. and Seeds, A.J. (2019, November). High Speed Photodetectors. In 2019 Asia Communications and Photonics Conference (ACP) (pp 1-3). IEEE.

Nagatsuma, T., Ducournau, G. and Renaud, C.C. (2016) Advances in terahertz communications accelerated by photonics. Nature Photonics, 10(6), pp.371-379. https://doi.
org/10.1038/nphoton.2016.65

\section{Personal Response}

\section{What would be the advantages of integrating UTC} into chip-scale devices?

II Key to the success of the technology is to prove its efficiency and in particular reduced energy
consumption. Chip-scale integration would enable the different components that are essential to the converter to be connected with lower losses thus optimising the energy efficiency while reducing size. One exciting devices on a silicon-based platform to produce a seamless electronic and photonic integrated microchip.

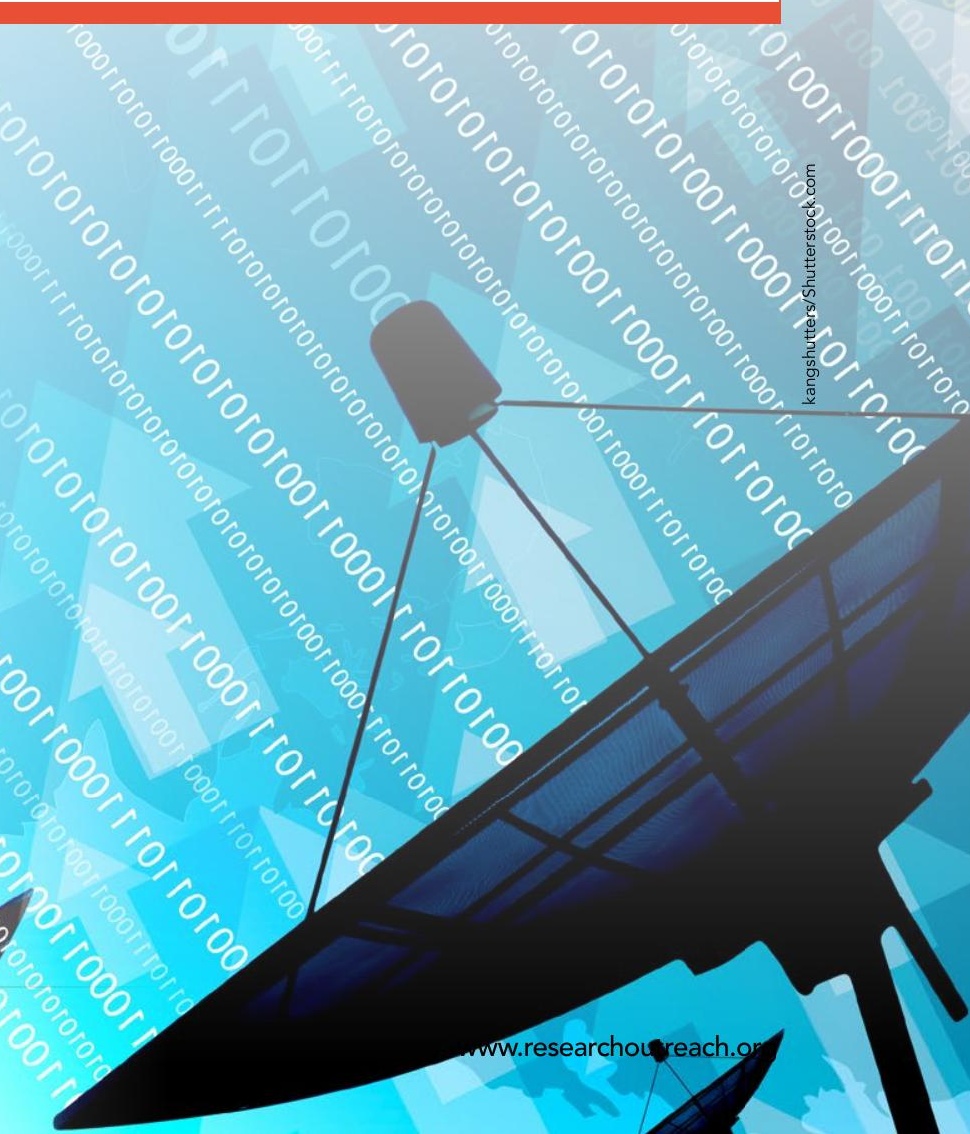

